\title{
Electric Spark Alloying of Radiant Coils for Pyrolysis Furnaces
}

\author{
Mikhail Taymarov ${ }^{1}$ and Elena Saltanaeva ${ }^{1, *}$ \\ ${ }^{1}$ Kazan State Power Engineering University, 420066 Krasnoselskaya st., 51, Kazan, Russian \\ Federation
}

\begin{abstract}
Currently, the petrochemical industry uses furnaces to produce ethylene, the main element of which is radiant coils designed for the decomposition of straight-run gasoline into pyrolysis gas, which is the main product for producing ethylene. In radiant coils, the gasoline decomposition process must take place at a temperature of about $800{ }^{\circ} \mathrm{C}$ with a high heating rate in order to avoid coking of the coils. Heat is supplied by radiation from the inner lining of the furnaces heated by the flame of flat-flame gas burners. For radiant heat transfer to occur, the surface of the coils must have a high degree of blackness. This article presents the developed technology for coating heating surfaces with shungite and the results of increasing their emissivity to intensify radiant heat transfer. Measurements of the emissivity after electrospark alloying were carried out by the radiation method, according to which, according to Kirchhoff's law, the emissivity is equal to the emissivity at equal temperatures.
\end{abstract}

\section{Introduction}

The heating surface of radiant coils in pyrolysis furnaces perceives heat due to radiation from the heated inner lining of furnaces, in which natural gas is burned in flat flame burners $[1,2]$. The main characteristic of the efficiency of radiant heat transfer for coils is their emissivity depending on temperature [3-7]. With an increase in the degree of emissivity, the amount of heat received by the radiant coils increases and the process of decomposition of straight-run gasoline vapors in them accelerates to obtain pyrolysis gas, which is then processed into ethylene. According to Kirchhoff's law [1-3], the emissivity is equal to the emissivity of the metal at equal temperatures and surface conditions. Therefore, to determine the degree of emissivity by the radiation method, the emissivity of the metal of the heating surfaces is investigated at equal temperatures. In experiments, it is customary to determine the integral normal emissivity of metal samples, since the difference between the hemispherical emissivity and the normal one lies within the experimental accuracy. The data on the emissivity of steels available in the scientific and reference literature are very limited and incomplete. As applied to pyrolysis furnaces, the monograph [3] gives the results on the normal integral degree of emissivity for $\mathrm{Cr} 15 \mathrm{Ni} 80$ steel without an alloy

\footnotetext{
*Corresponding author: elena_maister@mail.ru
} 
coating, oxidized at a temperature of $1273 \mathrm{~K}$ with an oxidation time in air of 1, 2, 4 and 20 hours. In other works, studies of the effect of shungite electrospark alloying coating were not carried out [8-10]. Consequently, further experimental studies of the directional degree of blackness of metals and, in particular, steels used in pyrolysis furnaces are relevant.

\section{Preparation of samples for research using electrospark alloying with shungite}

The electrospark alloying coating with a high degree of blackness is made of shungite from the Zazhoginsky deposit with a carbon content of an average of $30 \%$ by weight and being in an amorphous state in the form of fullerene. Fullerene is a special form of carbon due to the fact that the C60 fullerene molecule is an organic molecule, and the crystal formed by such molecules is called fullerite and is a link between organic and inorganic substances. The fullerite crystal has a density of $1.7 \mathrm{~g} / \mathrm{cm} 3$, which is significantly less than the density of graphite $(2.3 \mathrm{~g} / \mathrm{cm} 3)$ and diamond $(3.5 \mathrm{~g} / \mathrm{cm} 3)$. The shungite of the Zazhoginsky deposit contains carbon, on average $30 \%$ by weight in the form of fullerene and $57 \%$ silicon dioxide. With such a composition, the shungite of the Zazhoginsky deposit is a natural mineral that conducts an electric current. The electrical conductivity of shungite is $(1 \ldots 3) \times 103 \mathrm{sim} / \mathrm{m}$, the mechanical compressive strength is $1000 \ldots 1200 \mathrm{ktf} / \mathrm{cm} 3$, the thermal conductivity is $3.8 \mathrm{~W} /(\mathrm{m} \times \mathrm{K})$. The average value of the coefficient of thermal expansion in the temperature range $20 \ldots 600^{\circ} \mathrm{C}$ is $12 \times 10-61 / \mathrm{deg}$. The density of shungite is $2,1 \ldots 2,4 \mathrm{~g} / \mathrm{cm} 3$, the porosity is up to $5 \%$. The cost of shungite is $190 \mathrm{rubles} / \mathrm{kg}$. The Zazhoginskoe shungite deposit is located in the Medvezhyegorsk region of the Republic of Karelia, $5 \mathrm{~km}$ from the navigable bay of Lake Onega. The samples of metal, on which the electrospark alloying shungite coating was deposited, had dimensions of $50 \times 30 \times 10 \mathrm{~mm}$. Electrospark alloying with an electrode made of shungite from the Zazhoginsky deposit was carried out using an IL100-2 ultrasonic unit with a hand-held ultrasonic tool for fixing the electrode. As a result of alloying with a shungite electrode, the material of the anode electrode (shungite) is mechanically destroyed and secondary structures are formed in its working part. In this case, shungite erosion products are transferred to the coated part (cathode), on the surface of which processes associated with the diffusion of fullerene $C_{60}$ from the alloying material into the surface of the part take place. The resulting coating surface had a dark gray color with a metallic sheen. The power of the electric spark generator during coating was $180 \mathrm{~W}$. The thickness of the coating layer was $90 \mu \mathrm{m}$. Measurement of the coating thickness was carried out on a section under a MIM-8 microscope. The roughness of the outer surface of the coating was measured with a TR100 profilometer and amounted to $R a=6,5 \mu \mathrm{m}$ with a base length $S m=2,5 \mathrm{~mm}$.

\section{Procedure for measuring the integral emissivity of samples}

A schematic diagram of an experimental setup for measuring the emissivity of metal samples is shown in Fig. 1.

The flux of thermal radiation from the test sample 2 passes through the collimator tube 3 , is limited by the rotary diaphragm 4 , then is focused by the parabolic mirror 5 onto the infrared radiation detector 7 , which is used as a thermal column. The sample is heated to the required temperature using an electric resistance furnace 1 by changing the voltage applied to the furnace power supply. The electric furnace 1, together with the sample 2 and the limiting diaphragm, during the experiments, can be simultaneously rotated by the required angle $\theta$ relative to the optical axis. Shielding shutters 6 are used to determine the amount of background thermal radiation. Thermal stabilization of the infrared receiver is 
carried out by using a water-cooled housing. Maintaining a constant temperature of the circulating water in the receiver housing and in the collimator tube is performed using a thermostat.

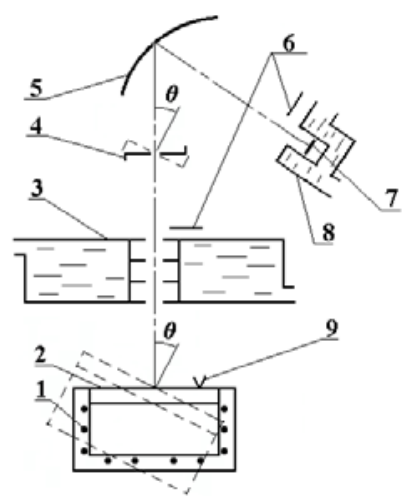

Fig. 1. Diagram of the experimental setup for measuring the directional emissivity $\varepsilon(\theta)$ :

1 - electric furnace for heating the samples; 2 - test sample; 3 - water-cooled collimator tube;

4 - rotary diaphragm; 5 - off-axis parabolic mirror; 6 - dampers (screens); 7 - receiver of infrared radiation; 8 - water-cooled housing; 9 - thermocouple.

The temperature of the emitting surface of the samples was measured by three chromelalumel thermocouples with a thermoelectrode diameter of $0,2 \mathrm{~mm}$. Thermocouples are spaced evenly around the circumference at a distance of $3 \mathrm{~mm}$ from the projection of the viewing angle of the radiation detector onto the sample. The thermocouple hot junctions are installed flush with the emitting surface of the samples.

Before starting the experiments, the infrared receiver is calibrated against a hightemperature standard emitter - a tubular absolutely black body (ABB), the effective blackness of which is $\varepsilon_{e b}=0,995$ [3].

The directional emissivity of the samples is determined by the formula:

$$
\varepsilon(\theta)=\varepsilon_{e b}\left(E(\theta)-E_{b}(\theta)\right) /\left(E_{0}(\theta)-E_{0 b}(\theta)\right),
$$

where $E(\theta), E_{0}(\theta)$ - respectively, the density of radiation from the test sample and the reference emitter at the same temperatures $T, \mathrm{~W} / \mathrm{m} 2 ; E_{b}(\theta), E_{0 b}(\theta)$ - background radiation density during measurements on a sample and a reference emitter, $\mathrm{W} / \mathrm{m} 2$.

In formula (1), the directional radiation density $E(\theta)$ is not only a characteristic of the diffuseness of radiation. In connection with the measurement scheme (Fig. 1), $E(\theta)$ is quantitatively reduced due to the diaphragm of the radiating metal area due to the rotary diaphragm.

If the rotary diaphragm 4 was not used, then when the sample 2 was rotated, the size of its emitting area would also increase. However, for a reference emitter - an absolutely black body - the radiation intensity is the same in all directions, since the emitting object is a cavity.

In addition to the direction of radiation, an important characteristic for the radiation of a metal is its roughness, the level of surface oxidation and, for heating conditions in air, the heating rate.

The following parameters are used to describe the surface roughness: $R a$ is the arithmetic mean deviation of the profile irregularities $(\mu \mathrm{m})$ and $S_{m}$ is the average step of the profile irregularities $(\mathrm{mm})$. 
In experiments to determine the emissivity $\varepsilon$, it is usually assumed that the temperature of the receiver $T_{r}$ is equal to the ambient temperature $T_{a m b}$ and, based on this, use the formula:

$$
\varepsilon=\left(T_{a b b}^{4}-T_{r}^{4}\right) /\left(T_{\text {sam }}^{4}-T_{r}^{4}\right),
$$

where $T_{\text {sam }}$ - is the sample temperature measured during the experiment using a thermocouple; $T_{a b b}$ - the absolutely black body temperature determined from the calibration curve based on the signal from the receiver.

When using formula (1), the radiation receiver is calibrated according to the temperature of the reference transmitter. However, it's not always in experiments that the $T_{r}$ is equal to $T_{a m b}$. Usually $T_{r}<T_{a m b}$ and formula (1) gives some errors in the results of experiments. The error can be estimated and reduced by introducing a correction for the temperature $T_{\text {corr }}$. The starting point for such an assessment is the heat balance equation at the receiver.

For the case of isotropically reflecting surfaces, the heat balance equation at the receiver can be written as:

$$
Q_{17}+Q_{h 7}+Q_{t}=0
$$

where $Q_{17}, Q_{h 7}$ - are the resulting heat fluxes of radiation between the sample 1 and the receiver 7 (Fig. 1), as well as between the housing of the receiver and the receiver 7; $Q_{t}-$ is the loss of thermal energy due to the thermal conductivity of the working ends of the receiver.

If the readings of the receiver are the same when sighting it first at the sample, then at the $\mathrm{ABB}$, then under the same measurement conditions, the values of $Q_{h 7}, Q_{t}$. Then:

$$
Q_{17}^{s a m}=Q_{17}^{a b b} .
$$

Equality (4) implies the equality of the incident flows:

$$
Q_{\text {inc } 17}^{\text {sam }}=Q_{\text {inc } 17}^{a b b},
$$

whence we obtain the equality of effective flows:

$$
Q_{\text {eff }}^{\text {sam }}=Q_{\text {eff }}^{a b b} .
$$

The effective radiation of the sample is the sum of its own radiation and the reflected radiation of the environment and the receiver housing. The effective radiation of ABB is equal only to its own. Considering the radiation of the surrounding bodies and the outgoing (Fig. 1) radiation of the receiver as black, and also using the equation of reciprocity and closedness of the angular coefficients [3], we can write:

$$
\begin{gathered}
Q_{\text {eff }}^{\text {sam }}=F_{1} \sigma_{0}\left\lfloor\varepsilon T_{\text {sam }}^{4}+(1-\varepsilon) \varphi_{16} T_{r}^{4}+(1-\varepsilon)\left(1-\varphi_{16}\right) T_{\text {amb }}^{4}\right\rfloor \\
Q_{\text {eff }}^{\text {sam }}=F_{1} \sigma_{0} T_{a b b}^{4},
\end{gathered}
$$

where $F_{1}$ - is the surface of the sample or $\mathrm{ABB}$, to which the receiver is sighted; $\varphi_{16}$ - is the integral angular coefficient of radiation from sample 1 to the receiver; $\sigma_{0}$ - is the Stefan-Boltzmann constant. Let's introduce the correction temperature: 


$$
T_{\text {corr }}^{4}=\varphi_{16} T_{r}^{4}+\left(1-\varphi_{16}\right) T_{a m b}^{4} .
$$

Transforming expressions (4-6), we obtain a formula for calculating the emissivity of a sample $\varepsilon$ in the form:

$$
\varepsilon=\left(T_{a b b}^{4}-T_{\text {corr }}^{4}\right) /\left(T_{\text {sam }}^{4}-T_{\text {corr }}^{4}\right) .
$$

With the emissivity of an absolutely black body $\varepsilon_{a b b} \neq 1$, the actual emissivity is:

$$
\varepsilon=\varepsilon_{a b b}\left(T_{a b b}^{4}-T_{c o r r}^{4}\right) /\left(T_{\text {sam }}^{4}-T_{\text {corr }}^{4}\right) .
$$

If the sample is located at a very small distance from the diaphragm, then $\varphi_{16}=1$, and $T_{\text {corr }}=T_{r}$. If the sample is located at a significant distance, then $\varphi_{16}=0$, and $T_{\text {corr }}=T_{a m b}$. The actual value of $T_{c o r r}$ is determined in the following order. The sample at room temperature is brought close to the radiation receiver without touching.

The value of the emissivity of the sample at room temperature $\varepsilon_{\text {room }}$ under these conditions is found by formula (10) from the condition that $T_{\text {corr }}=T_{r}$.

Then the sample is moved to a distance at which further experiments will be carried out to determine the emissivity of the samples.

With a new value of $T_{a b b}$ and a known $\varepsilon=\varepsilon_{\text {room }}$, according to formula (10), for each new sample, it is possible to find $T_{\text {corr }}$, which is considered constant during the experiment.

However, as experiments have shown, the value of the correction temperature has a noticeable effect at $T_{\text {sam }}>423 \mathrm{~K}$ and $\varepsilon \leq 0,1$.

When calibrating the receiver in energy units and the presence of background radiation, the emissivity of the sample $\varepsilon$ is determined by the more convenient formula:

$$
\varepsilon=\varepsilon_{a b b}\left(E-E_{a m b . b}\right) /\left(E_{a b b}-E_{a m b . b}\right),
$$

where $\varepsilon_{a b b}$ is the effective emissivity of the reference emitter - ABB; $E, E_{a b b}-$ respectively, the density of radiation fluxes from the test sample and the reference emitter at the same temperatures $T ; E_{a m b . b}$ - flux density of background ambient radiation.

\section{Calculation of experimental errors}

The largest errors in determining the emissivity are associated with temperature measurements using thermocouples. The general formula for determining the mean square error of temperature measurement using thermocouples can be written based on the average reduced values of partial errors in the form, $[1] \%$ :

$$
\sigma_{t}=1,1\left(\sigma_{1}^{2}+\sigma_{2}^{2}+\sigma_{3}^{2}+\sigma_{4}^{2}+\sigma_{5}^{2}+\sigma_{6}^{2}+\sigma_{7}^{2}\right)^{0,5},
$$

where $\sigma_{1}$ is the error caused by the inhomogeneity of thermoelectrode materials, $\pm(0,15$ $0,2) \% ; \sigma_{2}$ - thermocouple calibration error, $\pm(0,2-0,3) \% ; \sigma_{3}$ - the error of the secondary device [for the MPP-054 type millivoltmeter is $\pm(0,5-1) \%$, for the PP type potentiometer $\pm(0,2-0,5) \%$, for the V7-35 voltmeter $\pm 0,2 \%$; $\sigma_{4}$ - error in determining the resistance of the thermoelectric circuit of the thermocouple (when measured with a millivoltmeter), $\pm(1-1,2) \% ; \sigma_{5}$ - error in determining the temperature of the free ends, \pm 
$(0,2-0,3) \% ; \sigma_{6}-$ reading error on the scale of the secondary device, $\pm(0,5-0,8) \% ; \sigma_{7}-$ error of thermocouple setting $\pm(0,2-0,3) \%$.

Substituting the numerical values of the partial errors in formula (13), we obtain the value of the root-mean-square error in measuring the temperature using thermocouples $\sigma_{t}= \pm 1,8 \%$.

Determination of the emissivity of materials is a cumulative measurement and error of experiments, it includes the non-excluded systematic error of the method and measuring instruments.

The systematic error in determining the integral emissivity is written as:

$$
\sigma_{i}=1,1\left(\sigma_{i . f}^{2}+\sigma_{\text {rough }}^{2}+\sigma_{\text {reg }}^{2}+\sigma_{P}^{2}+\sigma_{m}^{2}\right)^{0,5},
$$

where $\sigma_{i . f}$ is the systematic error in determining the ratio of the integral radiation fluxes of the test sample and absolutely black body; $\sigma_{\text {rough }}$ - is the error in measuring the roughness parameters; $\sigma_{r e g}$ - error of registration of the signal from the radiation receiver; $\sigma_{P^{-}}$ error in processing graphic results; $\sigma_{m}$ - methodical error.

The systematic error in determining the ratio of the integral radiation fluxes is written in the form:

$$
\sigma_{i . f}=1,1\left(\sigma_{E}^{2}+\sigma_{E 0}^{2}\right)^{0,5}
$$

where $\sigma_{E}, \sigma_{E 0}$ are, respectively, systematic errors in determining the integrated radiation energy density of the sample under study and an absolutely black body, defined as:

$$
\sigma_{E}, \sigma_{E 0}=1,1\left(4 \sigma_{t, t 0}^{2}+\sigma_{r e g}^{2}+\sigma_{P}^{2}\right)^{0,5}
$$

where $\sigma_{t}, \sigma_{t 0}$ are, respectively, the errors in measuring the temperature of the emitting surface of the sample and the cavity of an absolutely black body.

When processing the experimental data, the average of the arithmetic measurements is taken as the measurement result $a_{a v}$.

The estimate of the standard deviation $S$ is described by the expression:

$$
S=\left[\sum\left(x_{i}-a_{a v}\right)^{2} / n(n-1)\right]^{0,5},
$$

where $n, x_{i}$ are number and result of measurements. The summation is carried out from 1 to $n$.

In accordance with the value of the coefficient of reliability $\alpha=0,95$ and the number of measurements $n$, the Student's coefficient $t_{\alpha}$ is determined. In this case, the formula for calculating the random error is written as follows: $\Delta a=t_{\alpha} S$.

Hence the result with the boundaries of the random error: $a=a_{a v} \pm \Delta a$ and the relative random error $\xi=(\Delta a / a) 100 \%$.

The total measurement error is defined as a set of random and non-excluded systematic errors:

$$
\delta=1,1\left(\xi^{2}+\sigma^{2}\right)^{0,5}
$$


Since $\sigma / \xi>8$ was obtained in experiments on the study of the emissivity of materials, the random error $\xi$ can be neglected. The error limits of the measurement results can be estimated by the non-excluded systematic error $\pm \sigma(\%)$.

Systematic error in determining the integral emissivity of materials at $1000 \mathrm{~K}$ $\sigma_{i}= \pm 8,2 \%$ (error components $\pm: \sigma_{t}=1,8 \% ; \sigma_{t 0}=1,8 \% ; \sigma_{P}=0,8 \% ; \sigma_{\text {reg }}=0,8 \%$; $\left.\sigma_{E}=4,2 \% ; \sigma_{E 0}=4,2 \% ; \sigma_{i . f}=6,5 \% ; \sigma_{\text {rough }}=1,1 \% ; \sigma_{m}=3,2 \%\right)$.

The largest errors are associated with measuring the temperature of the emitting surface of the sample and the cavity of an absolutely black body. The methodological error $\sigma_{m}= \pm 3,2 \%$ includes the error in determining the content of the main chemical components, the value of which is taken according to the recommendations [1]

\section{Results obtained and their discussion}

The results of measurements of the integral normal emissivity of metal samples before and after electrospark alloying with shungite are given in table 1 .

Table 1. Integral normal emissivity of steels alloyed with shungite $\varepsilon_{n}$ at different temperatures $T$ when heated in air with a speed $V=0,042(\mathrm{deg} / \mathrm{s})$

\begin{tabular}{|c|c|c|c|c|c|}
\hline \multirow[t]{2}{*}{ Sample type } & \multirow[t]{2}{*}{ Steel grade } & \multicolumn{4}{|c|}{$\varepsilon_{n}$ at temperature $T, K$} \\
\hline & & 600 & 700 & 800 & 900 \\
\hline After alloying & \multirow{2}{*}{$12 \mathrm{CrMoV}$} & 0,88 & 0,91 & 0,93 & 0,95 \\
\hline Before alloying & & 0,50 & 0,70 & 0,83 & 0,85 \\
\hline After alloying & \multirow{2}{*}{ Cr23Ni32Ti } & 0,86 & 0,87 & 0,89 & 0,93 \\
\hline Before alloying & & 0,78 & 0,81 & 0,84 & 0,87 \\
\hline After alloying & \multirow{2}{*}{ 08Cr18Ni10Ti } & 0,63 & 0,64 & 0,66 & 0,69 \\
\hline Before alloying & & 0,53 & 0,55 & 0,57 & 0,60 \\
\hline
\end{tabular}

As can be seen from Table 1, electrospark alloying of steels with shungite increases their emissivity by an average of $12 \%$. It should be borne in mind that the surface layer of the metal doped with shungite is dense and does not undergo oxidation with the formation of an oxide film, which increases the thermal resistance of radiant coils and slows down the heating process of the technological product - straight-run gasoline vapor. A slowdown in the heating process leads to increased coking of the radiant coils and the premature need to clean them.

\section{References}

1. V.I. Trembovlya, Thermal testing of boiler plants. Moscow: Energiya, pp.297 (2007)

2. W. Pepperhof, Archiven Eisenhuttenwerken. Band 9. No. 10. pp.12-16 (2012)

3. A.G. Blokh, Heat transfer by radiation. M.: Energoatomizdat, pp.432 (1991)

4. M.A. Taimarov, Intensification of thermal decomposition of straight-run gasoline in pyrolysis furnaces using acoustic burners. Vestnik of Kazan technological University v.18, No. 22, pp.59-62 (2015)

5. M.A. Taimarov, M.R. Sharipov, A.R. Khaertdinova, P.G. Akparsov, A device for superheating a steam-water gas mixture. Vestnik of Kazan technological University, T.17, N 10, pp.185-187 (2014) 
6. Zhang Zhaobing, Cracking furnace CBL. Patent for invention RU No. 2552417 dated 06/10/2015

7. M.A. Taimarov, E.A. Saltanaeva, L.I. Kasimova, Radiation characteristics of the flame in the furnaces of boilers when burning high-water-cut fuel oil. Bulletin of Kazan State Power Engineering University. Vol. 11, No. 1 (41), pp. 85-93 (2019)

8. S. Mayo, Slurry Phase Hydrocracking: Bottoms Upgrading for Today's Market. Chemical Engineering World, March, pp. 52-59 (2014)

9. Shi Shidong, Jin Jiadi. Proc. Int. Symp. on Clean Coal Technology, Xiamen, China, pp. 500-505 (2017)

10. X. Kechang, L. Wenying, Z. Wei, Proc. Int. Conf. Coal Science and Technology, Nottingam, UK, August, pp. 234-245 (2007). 